A review article

\title{
Vitamin D deficiency is less prevalent among children with common medical illnesses than apparently healthy children in sub-Saharan Africa: A systematic review and meta-analysis
}

\author{
Mohammed Feyisso Shaka ${ }^{1 *}$ Robel Hussen kabthymer ${ }^{1}$, Meiraf Daniel Meshesha ${ }^{2}$ \\ ${ }^{1}$ Dilla University, College of Health and Medical Sciences, School of Public Health, Dilla, \\ Ethiopia robelhussen@rocketmail.com \\ ${ }^{2}$ Dilla University, College of Health and Medical Sciences, School of Medicine, Dilla, \\ Ethiopia \\ *corresponding author- Mohammed Feyisso Shaka (MF) \\ Email: mamfys8@gmail.com
}

Background: Studies shows that 25(OH)D status appears to have some beneficial influence on the incidence and severity of some types of infections. However, studies with vitamin D supplementation on young children produced conflicting results. Method: A systematic review of PubMed, CINAHL, Web of science, global health and Google scholar electronic databases was conducted. STATA Version 14 was used for analysis. Heterogeneity of studies was assessed using $\mathrm{I}^{2}$ test. A random-effects model was used to estimate the pooled prevalence among both healthy and sick children. Result: A total of 1212 articles were retrieved from data bases of which 13 papers were included. The pooled prevalence of vitamin D deficiency among healthy children was $50.06 \%$ with mean serum vitamin D level of $41.06 \mathrm{~nm} / \mathrm{L}$. The pooled prevalence among the sick children was $39.36 \%$ with $66.96 \mathrm{~nm} / \mathrm{L}$ of mean concentration. The pooled prevalence among healthy children was significantly higher compared to those who have common medical illnesses and the pooled mean concentration among the sick was also much higher than the mean concentration among healthy children. Conclusion: The pooled prevalence among both group of population was significantly of public health concern and the prevalence among healthy children was much higher among sick children.

Key words: Vitamin D; under five; healthy; sick; sub-Saharan

\section{Introduction}

Micronutrients deficiencies in children can have multi-dimensional health impact on growth, immune system, cognitive development and physical growth and development[1]. Its deficiencies are also widely found in low income countries where micronutrient deficiencies account for approximately 7 percent of the global disease burden[2].

Globally, an estimated two billion people suffer from a chronic deficiency of essential vitamins and minerals (micronutrients), a condition known as hidden hunger[2] with Vitamin 
D deficiency being among the common micronutrient deficiencies worldwide[3]. According to the recent estimates, greater than $50 \%$ population is at risk in the globe[4].

As general consensus, serum 25-hydroxyvitamin D (25(OH)D) level below $50 \mathrm{nmol} / \mathrm{L}$ considered as a cutoff point for vitamin D deficiency and if its serum level is less than 25 $\mathrm{nmol} / \mathrm{L}$, severe deficiency is diagnosed[3]. Vitamin D deficiency is associated with infection and increased autoimmunity[5]. Different studies among under five children shows that decreased level of $25(\mathrm{OH}) \mathrm{D}$ were more prevalent among under five children with RTIs and its deficiency was associated with increased risk of RTIs. Furthermore, studies also shows that, normal to high serum $25(\mathrm{OH}) \mathrm{D}$ status appears to have some beneficial influence on the incidence and severity of some, though not all, types of infections. However, studies with vitamin D supplementation on

young children produced conflicting results, and it still remains unclear whether supplementation may be of benefit and at what doses[6].

An observational study conducted to assess the serum concentration of $25(\mathrm{OH}) \mathrm{D}$ among children with active TB using non-TB pneumonia and healthy controls revealed that larger number of vitamin D deficient children were from active TB group[7]. Similarly, many studies conducted elsewhere[8-12] delineated similar findings including Dabla's study conducted among pediatric osteoarticular tuberculosis patients which revealed that $56 \%$ of active TB cases were vitamin D deficient and had lower levels of serum 25(OH)D compared to healthy controls[9]. However, significant number of studies found no significant difference in vitamin D deficiency status TB patients and healthy children[13-15].

For growing children, deficiency of Vitamin D is among the issues of significant concern while there are limited information on its association with malnutrition[16,17]. Studies shows that, linear growth could be affected by vitamin D status of children even in the absence of rickets clinical signs[18]. From the evidences currently available in literature world, a strong evidence base of the contributions of vitamin D deficiency for malnutrition, both regionally and globally, has been lacking. Even though the role of macronutrients in physical growth is well known, the roles of micronutrients including vitamin D in physical growth is not well 
established. Particularly, evidences with respect to the role of vitamin D on stunting among children are not conclusive, and sometimes conflicting [16-21].

Hence, these review was done to avail better data on the relation between common medical illnesses and micronutrient deficiency particularly vitamin $\mathrm{D}$, to guide intervention programs and to prioritize global focus on addressing vitamin D deficiency.

\section{Materials and Methods}

\section{Study design and search strategy}

A systematic review and meta-analysis study was conducted to determine and compare the pooled prevalence of vitamin D deficiency among apparently healthy and sick under five children in sub-Saharan Africa. The major databases of PubMed, Cochrane library, CINAHL and Google scholar were used for systematic search of literatures with Boolean operators like “AND", "NOT" and "OR". Using the Boolean operator we searched as: ((vitamin d"[MeSH Terms] OR "ergocalciferols"[MeSH Terms] OR vitamin D [Text Word])) AND ("child"[MeSH Terms] OR CHILDREN[Text Word])) AND "Africa South of the Sahara"[Mesh])). A search output from different data bases were shown below (Table 1).

Table 1: Summary of search results for the PubMed, Google Scholar and other databases to assess the prevalence of vitamin D deficiency among under five children in Sub-Saharan Africa.

\begin{tabular}{|l|l|l|}
\hline Databases & Searching terms & $\begin{array}{l}\text { Number of } \\
\text { studies }\end{array}$ \\
\hline Google scholar & All in title: vitamin D OR ergocalciferols AND Children & 852 \\
\hline PubMed & $\begin{array}{l}\text { Search (("vitamin d"[MeSH Terms] OR } \\
\text { "ergocalciferols"[MeSH Terms] OR vitamin D[Text Word])) }\end{array}$ & 331 \\
& $\begin{array}{l}\text { AND ("child"[MeSH Terms] OR CHILDREN[Text Word] })) \\
\text { AND "Africa South of the Sahara"[Mesh] }\end{array}$ & \\
\hline Web of science, CINAHL "prevalence" or "magnitude" and "Vitamin D" and \\
and global health & $\begin{array}{l}\text { "Deficiency" or "level" and "children" or "Child" or } \\
\text { "ergocalceferols" and "sub-Saharan Africa" }\end{array}$ & \\
\hline
\end{tabular}




\begin{tabular}{|l|l|l|}
\hline Total retrieved articles & 1212 \\
\hline $\begin{array}{l}\text { Full text available papers } \\
\text { appropriate to our review }\end{array}$ & & 9 \\
\hline
\end{tabular}

\section{Selection of study and eligibility criteria}

All studies that were conducted on vitamin D deficiency among under five children and those fulfilled the inclusion criteria according to the interest of the study were included. All types of article types published in English were included. Initially, availability of full text titles, and abstracts of the articles were assessed. Then the full papers of relevant articles were reviewed. We excluded articles with inaccessible full paper.

\section{Inclusion criteria}

Study area: studies conducted in Sub-Saharan Africa only.

Study design: observational studies (cross-sectional, case-control and cohort studies)

Outcome measures: Studies that measured 25(OH)D in blood

Language: studies published/written in the English language only were included

Population: studies conducted among under five children.

Publication issue: both published and unpublished articles were searched

Study period: $2010-2020$

\section{General characteristics of studies}

In the first step screening, we got a total number of 1212 papers and 478 papers were excluded due to repetition. According to the titles and abstract 621 papers were excluded. Thirteen papers were found to be suitable. But the full text of 1 paper was inaccessible and after assessing the full text 3 papers were excluded due to failure to report outcome of interest/exposure and 9 papers were finally included in the study (Fig.1). After identification of the available papers, the papers were categorized in to two (healthy and sick) based on the type of population used to study. If the individual study used both types of population we used the included population of each population to do the pooled measurement for the current study.

1112 Records identified through database searching

pubmed $(\mathrm{n}=331)$, Google scholar $(\mathrm{n}=852)$ 


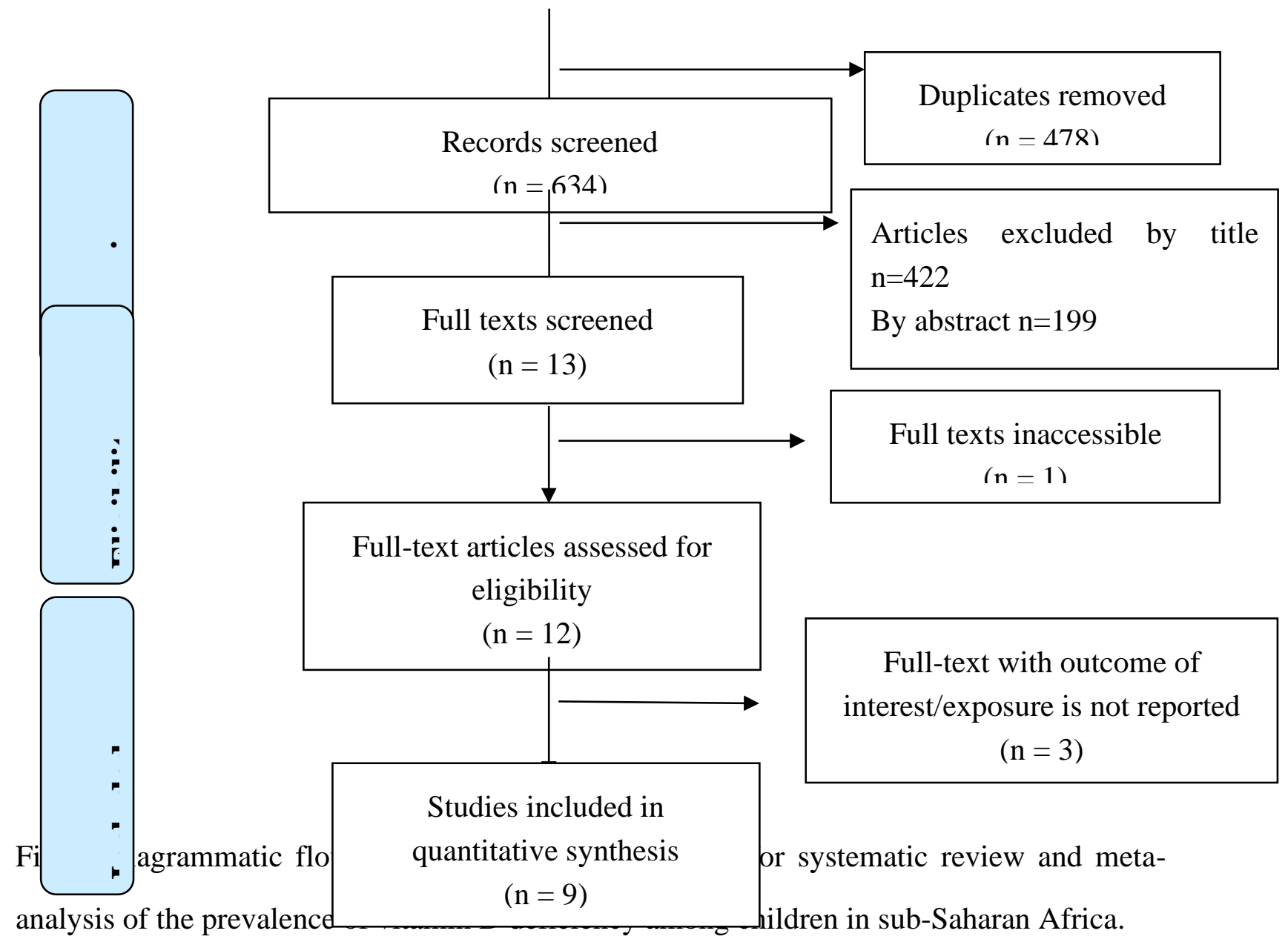

\section{Data extraction}

Data extraction for all the necessary data was done by RH and MF independently using a standardized data extraction format prepared in Microsoft Excel. Any disagreements between the authors during data extractions was discussed and reached on consensus. The data extraction format includes first author, publication year, name of country, sample size, included and number of event and prevalence with $95 \% \mathrm{CI}$. The main outcome of interest for this study is prevalence of vitamin D deficiency among healthy and sick children in subSaharan Africa. The main types of illnesses used in this study were TB, malnutrition and HIV/AIDS.

\section{Risk of bias}

The two authors (RH and MF) independently assessed the risk of bias for each article using the Newcastle-Ottawa Scale quality assessment tool[22]. The tool has indicators consisting 
of three main parts in which, the first part has five components and it assesses the methodological quality of each study. The second part of the assessment tool examines the comparability of the studies. The last part of the tool measures the quality of the original articles with respect to their statistical analysis. Using an assessment tool as a guideline, two authors independently evaluated the qualities of the original articles. Quality of each study was evaluated using these parameters; those with medium (fulfilling $50 \%$ of quality assessment criteria) and high quality ( $>=6$ out of 10 scales) were included for analysis. Disagreements between assessors were solved by taking the mean score of their assessment results.

\section{Data processing and analysis}

After extraction, the data were imported to STATA Version 14.0 statistical software for analysis. The standard error for each original study was calculated using the binomial distribution formula. Heterogeneity among reported prevalence was assessed by computing p-value $\mathrm{I}^{2}$ test static[23]. As the test statistic showed there is significant heterogeneity among the studies $\left(\mathrm{I}^{2}=98.3 \%, \mathrm{p}=0.000\right)$ and as a result a random effect meta-analysis model was used to estimate the pooled effect. To reduce the random effect variations between the point estimates of the primary studies, a subgroup analysis was done based on sample size. Funnel plot and Egger's test at 5\% significance level was used to asses the publication bias. From the result, the articles were symmetrically distributed and Egger's test at 5\% significant level was not significant for the publication bias. Pooled prevalence with $95 \%$ confidence intervals was presented in the forest plot format for both healthy and sick children. For reporting the finding 'Preferred Reporting Items for Systematic Reviews and Meta-Analyses (PRISMA)' protocol was used

\section{Results}

\section{Prevalence of vitamin D deficiency among healthy children}

The finding from the studies included for assessment of the pooled prevalence of vitamin D deficiency among healthy children revealed that, the prevalence was $50.06 \%$ (CI ranges from $33.46 \%$ to $66.67 \%$ ) (Fig. 2). On the other hand, sub-group analysis was done using sample size of the included studies as unit of sub-grouping. Accordingly, the pulled prevalence was 
$52.18 \%$ with CI of $32.78 \%$ to $71.57 \%$ among those having less than 100 sample size and $46.51 \%$ with CI of 4.44 to 97.31 among those having less than 100 sample size (Fig. 4). The pooled mean serum vitamin D level among the healthy children was $41.06 \mathrm{~nm} / \mathrm{L}$ with CI of $23.82 \mathrm{~nm} / \mathrm{L}$ to $58.31 \mathrm{~nm} / \mathrm{L}$ (Fig. 4 ).

Study

ID

$\mathrm{ES}(95 \% \mathrm{Cl})$

\begin{tabular}{|c|c|c|c|}
\hline RWEBEMBERA et al (2013) & $\rightarrow$ & & $34.55(27.81,41.29)$ \\
\hline sudfeld et al (2014) & & - & $60.97(57.86,64.08)$ \\
\hline ludmir et al (2016) & $\longrightarrow$ & & $17.00(5.50,28.50)$ \\
\hline sudfeld et al (2017) & & + & $76.40(72.95,79.85)$ \\
\hline Hassam et al (2019) & $\longrightarrow$ & & $31.90(18.57,45.23)$ \\
\hline Bellows et al (2017) & 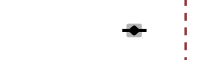 & & $36.40(33.13,39.67)$ \\
\hline Bodin et al (2019) & & $\rightarrow$ & $90.00(83.97,96.03)$ \\
\hline Overall $(\mathrm{I}$-squared $=98.8 \%, \mathrm{p}=0.000)$ & & & $50.06(33.46,66.67)$ \\
\hline NOTE: Weights are from random effects analysis & & & \\
\hline & & 10 & 00 \\
\hline
\end{tabular}

Figure: 2 Pooled prevalence of vitamin D deficiency among healthy children in subSaharan Africa

\section{Publication bias using funnel plot for prevalence among healthy children}

Funnel plot and Egger's test were used to test Publication bias. From the result the articles were symmetrically distributed (Fig. 3) and Egger's test result for small study effects revealed that publication bias in estimating vitamin D deficiency was not statistically significant. 
Funnel plot with pseudo 95\% confidence limits

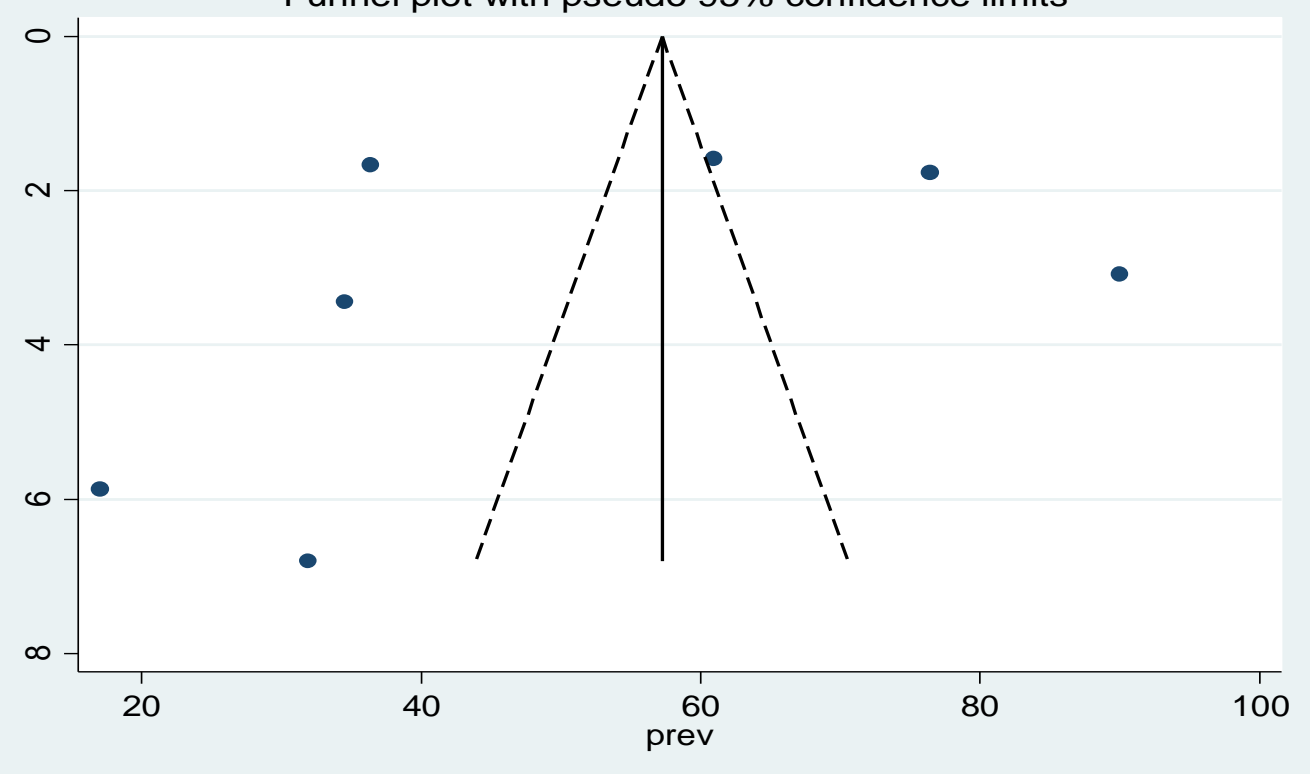

Figure 3: Funnel plot with $95 \%$ confidence interval of the pooled prevalence of vitamin D deficiency among healthy under five children in sub-Saharan Africa, 2020

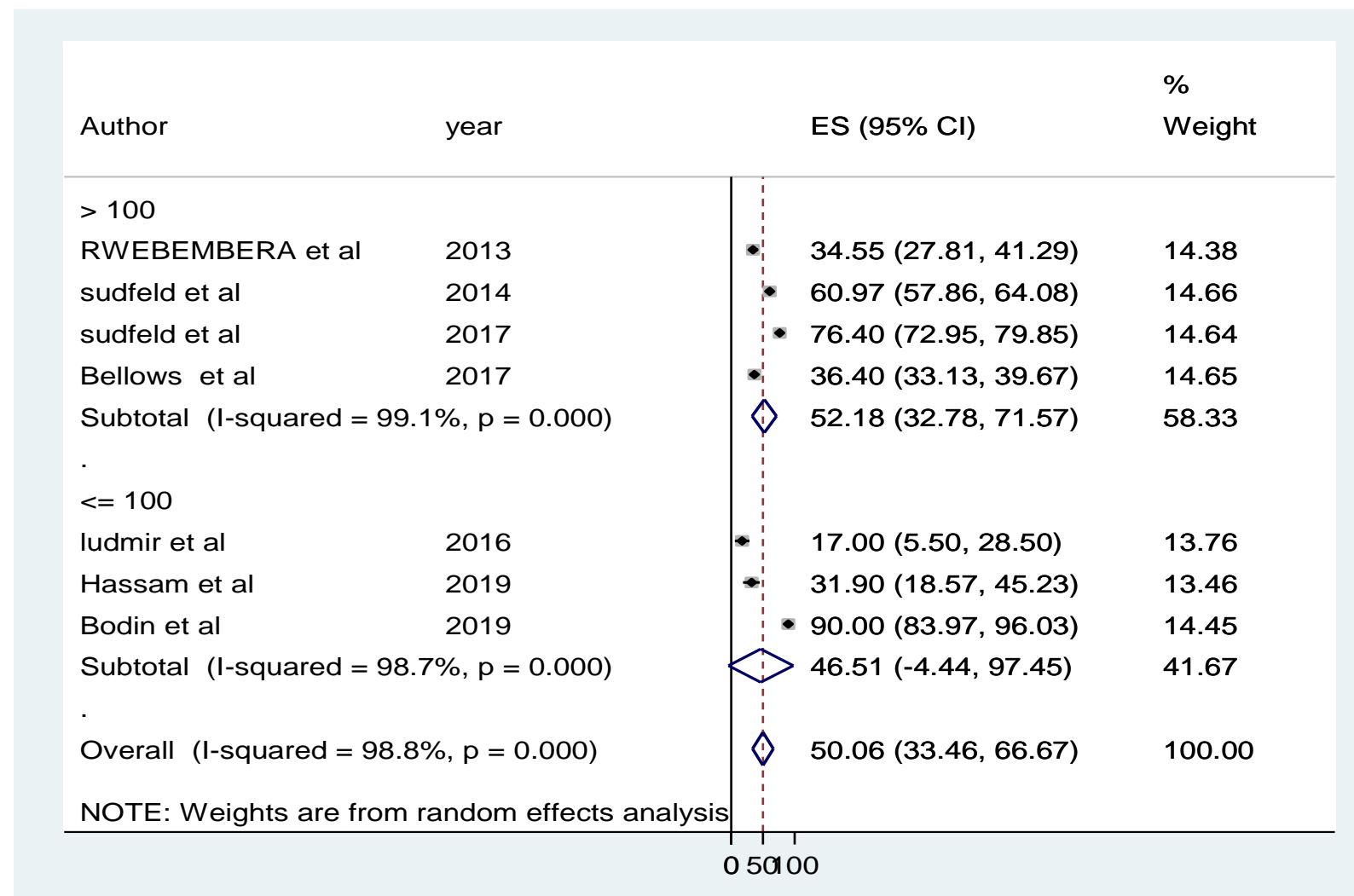

Figure 4: Subgroup analysis (using sample size) of pooled prevalence of vitamin D deficiency among healthy children in sub-Saharan Africa 
Study

ID

ES $(95 \% \mathrm{Cl})$

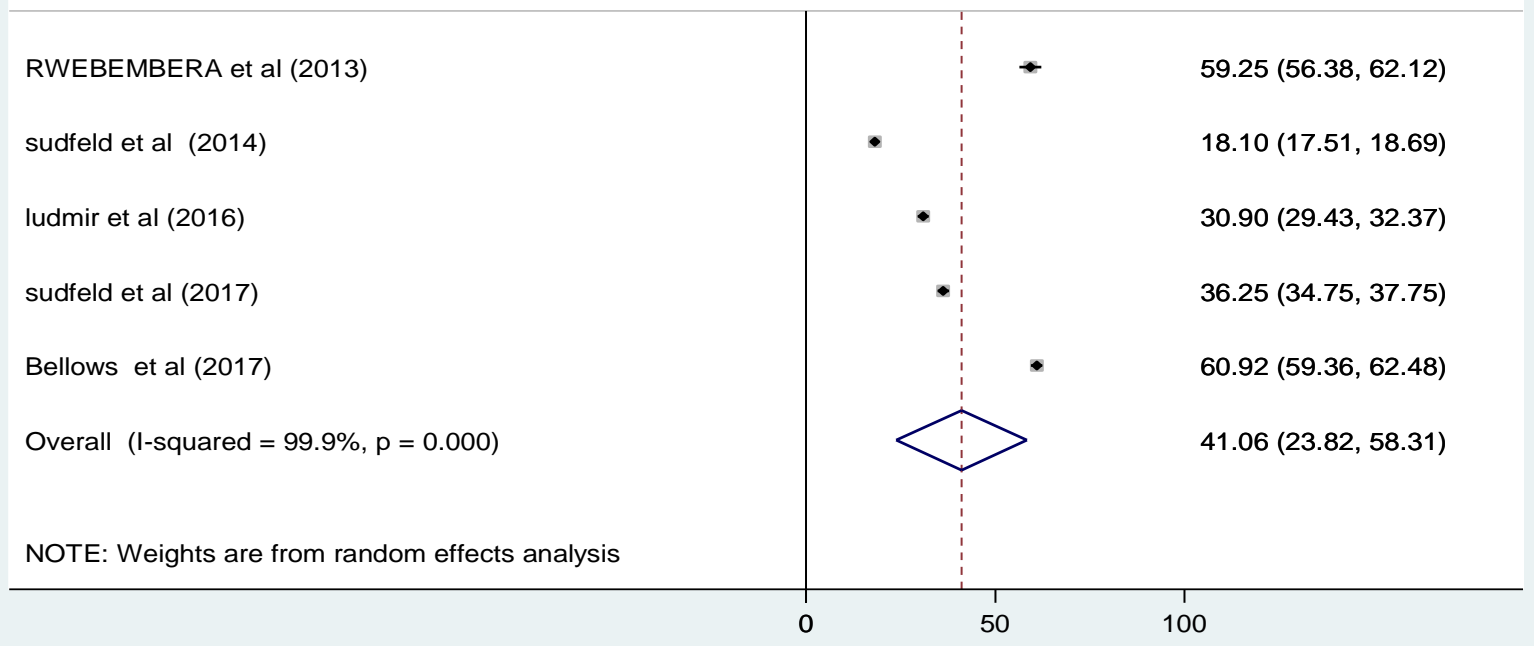

Figure 5: Mean serum vitamin D level among healthy children in sub-Saharan Africa,

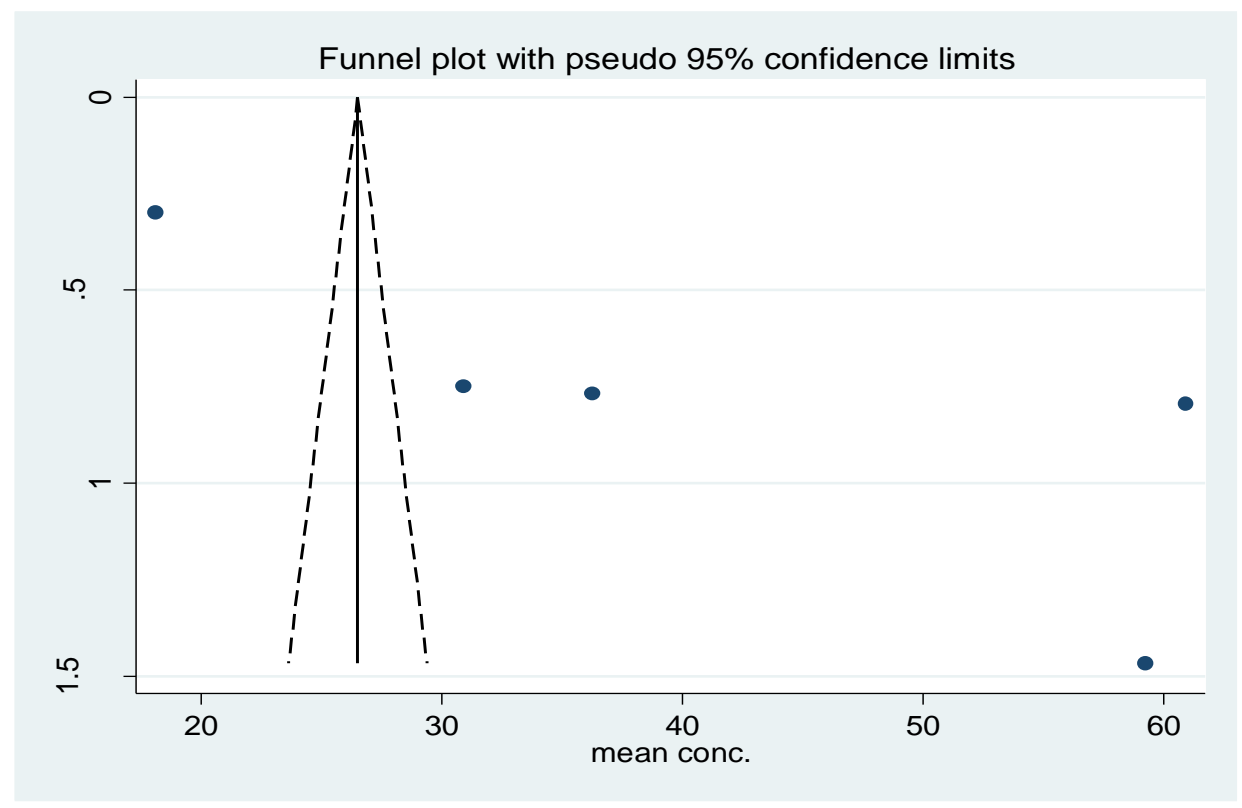

Figure 6: Publication bias for mean concentration of serum vitamin D among health children in sub-Saharan Africa

\section{Pooled prevalence of vitamin D deficiency among sick children in sub-Saharan Africa}

Analysis was done to assess the pooled prevalence of vitamin D deficiency among sick children (children having certain medical conditions). The analysis result revealed that, the 
pooled prevalence of vitamin D deficiency among the sick children was $39.36 \%$ with CI of 20.57 to $57.96 \%$ (Fig. 7). The pooled mean serum vitamin D level among the sick children was $66.96 \mathrm{~nm} / \mathrm{L}$ with $95 \% \mathrm{CI}$ of $54.81 \mathrm{~nm} / \mathrm{L}$ to $79.11 \mathrm{~nm} / \mathrm{L}$ (Fig. 8). Publication bias was assessed using funnel plot and the diagram shows that, there is symmetrical distribution of articles showing no significant publication bias (Fig. 9).

Study

ID

ES $(95 \% \mathrm{Cl})$

Sudfeld et al (2014)

ludmir et al (2016)

Hassam et al (2019)

Nabeta et al (2015)

Walli et al (2017)

Hassam et al (2019)

Overall $(\mathrm{I}$-squared $=96.6 \%, p=0.000)$

NOTE: Weights are from random effects analysis

0

50

100

$56.92(50.82,63.02)$

$21.00(8.22,33.78)$

$70.20(60.95,79.45)$

$14.60(8.20,21.00)$

$30.60(22.80,38.40)$

$42.60(28.46,56.74)$

$39.36(20.77,57.96)$

Figure 7: Pooled prevalence of vitamin D deficiency among sick children in sub-Saharan Africa 
Funnel plot with pseudo 95\% confidence limits

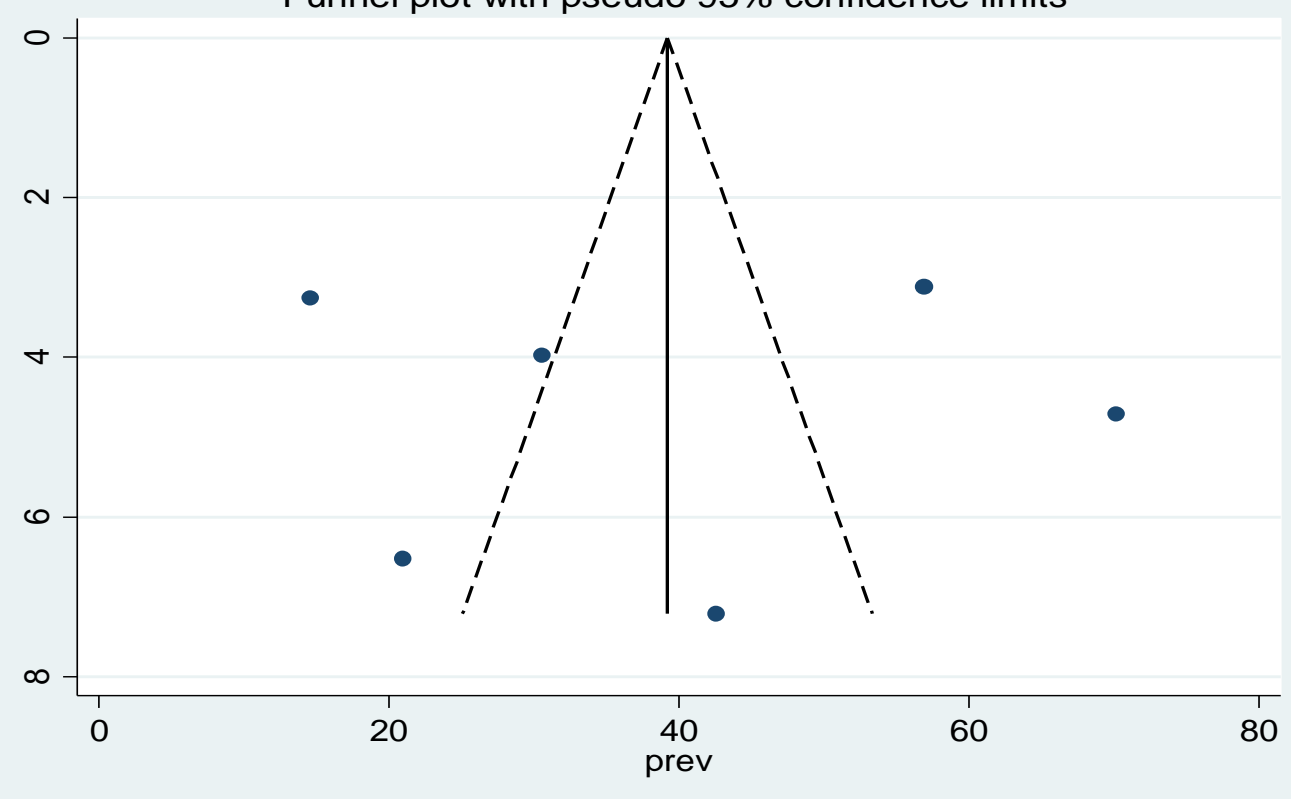

Figure 8: Funnel plot with $95 \%$ confidence interval for publication bias of prevalence of vitamin D deficiency among sick under five children in sub-Saharan Africa, 2020

Study

ID

ES $(95 \% \mathrm{Cl})$

Sudfeld et al (2014)

ludmir et al (2016)

Nabeta et al (2015)

Walli et al (2017)

Ahmed et al (2014)

Jones et al (2017)

Overall $(\mathrm{I}$-squared $=98.3 \%, \mathrm{p}=0.000$ )

NOTE: Weights are from random effects analysis

$0 \quad 50$

46.50 (43.33, 49.67)

- $\quad 80.50(76.88,84.12)$

$\rightarrow \quad 81.25(75.81,86.69)$

- $\quad 74.20(71.59,76.81)$

$61.50(54.35,68.65)$

$57.80(53.21,62.39)$

$66.96(54.81,79.11)$

Figure 9: Mean serum vitamin D level among sick children in sub-Saharan Africa 


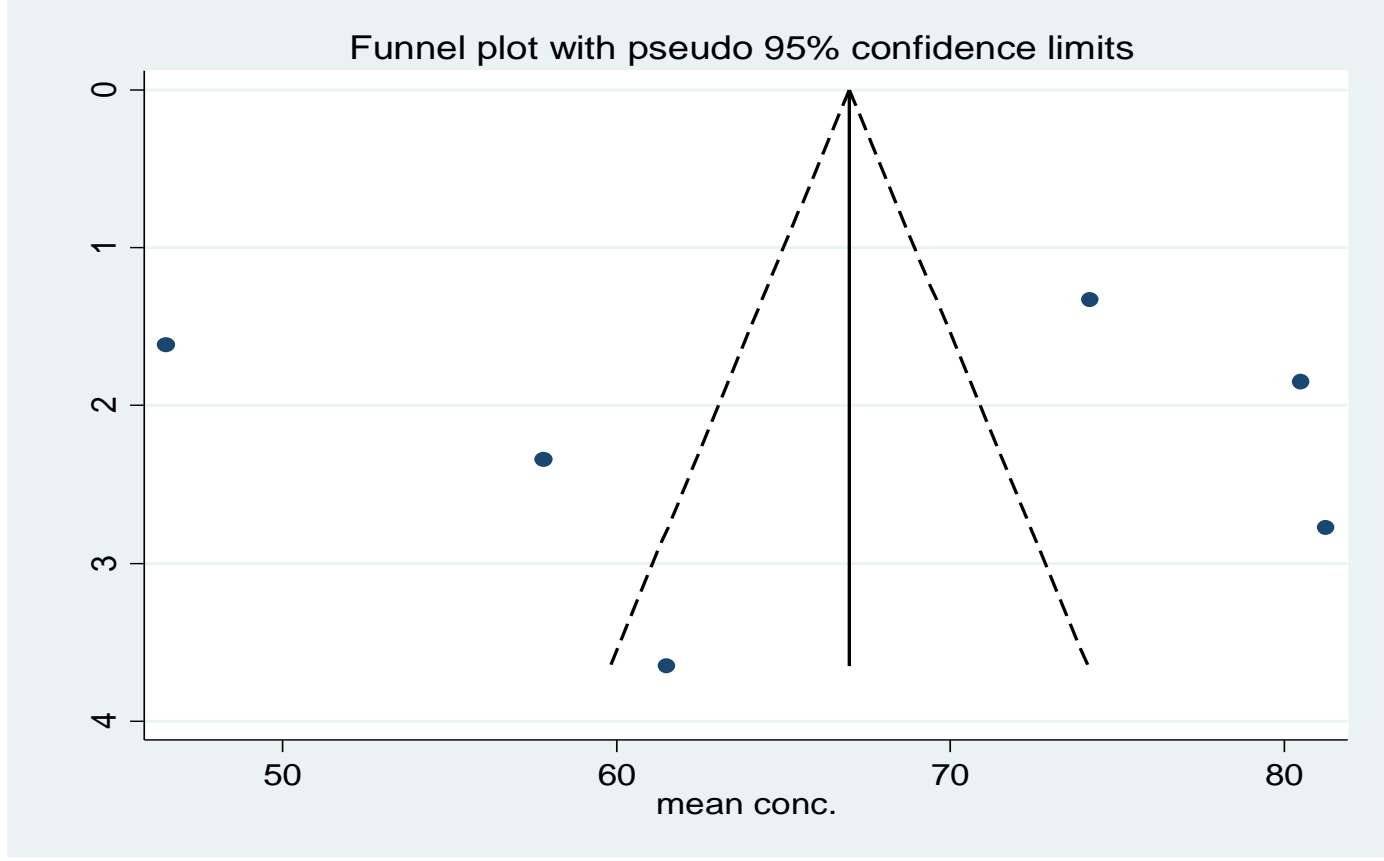

Figure 10: Publication bias for mean concentration of serum vitamin D among sick children in sub-Saharan Africa, August 2020

Subgroup analysis result among sick children using sample size as unit of subgrouping Subgroup analysis was done to assess the prevalence of vitamin D deficiency among children using sample size as unit of subgroup analysis and the result revealed that prevalence of vitamin D deficiency was $34.07 \%$ with CI of 8.05 to 60.08 among those having greater than 100 number of participants and $44.85 \%$ with CI of 14.41 to 75.30 among those having less than 100 number of participants. 


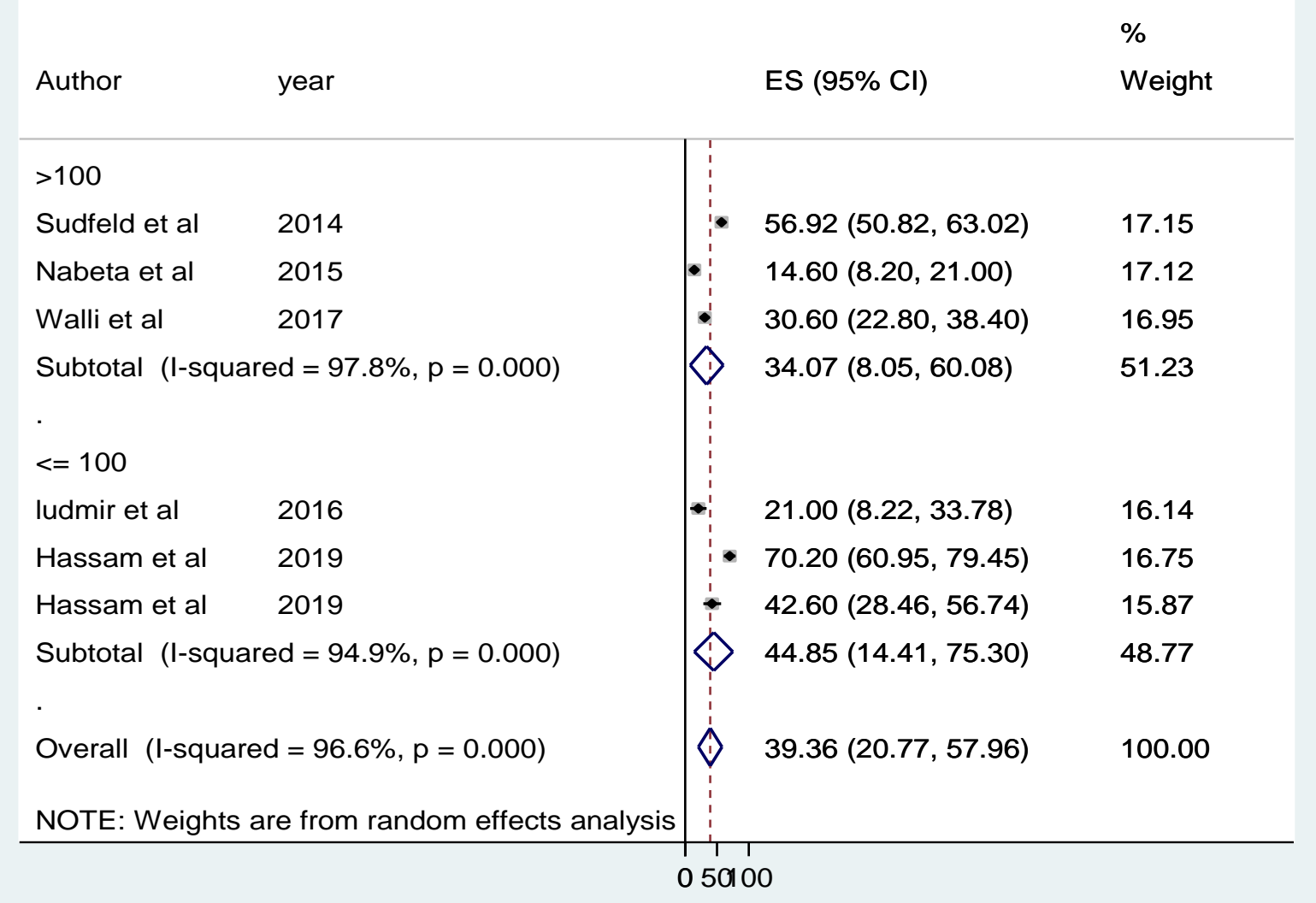

Figure 11: Subgroup analysis (using sample size) of pooled prevalence of vitamin D deficiency among healthy children in sub-Saharan Africa

\section{Discussion}

This study has tried to assess the pooled prevalence of vitamin D deficiency among under five children in sub-Saharan Africa. Attempts were made to see the pooled prevalence among the healthy and sick children separately. The common illness conditions considered in this review were mainly malnutrition, HIV/AIDS and tuberculosis.

From the result of analysis, the pooled prevalence among healthy children in sub-Saharan Africa was found to be $50.06 \%$ with $95 \%$ CI ranging from $33.46 \%$ to $66.67 \%$. On the other hand the pooled prevalence among sick children was $39.36 \%$ with CI of 20.57 to $57.96 \%$. Among the studies included for the sick children the lowest prevalence (14.6\%) of vitamin D deficiency was found in Uganda among malnourished children[24] and the highest prevalence (70.2\%) was from Tanzania among patients with diarrhea[25]. Regarding the healthy children the lowest prevalence (17\%) was among children from Botswana[26] and the highest prevalence of $76.4 \%$ was from Tanzania[27]. In this study the pooled prevalence 
of vitamin D deficiency among both healthy and sick children was found to be significantly of public health concern.

Regarding the comparison between the pooled prevalence among the sick and healthy children, the pooled prevalence among healthy children was found to be significantly higher. Correspondingly, the mean serum vitamin D level among healthy children $(41.06 \mathrm{~nm} / \mathrm{L})$ was found to be significantly lower than the level among sick children $(66.96 \mathrm{~nm} / \mathrm{L})$. The finding of the current study was in contrary to the presumed expectations and findings of other individual studies conducted elsewhere which shows vitamin D deficiency more prevalent among people with some type illnesses and health conditions[28,29]. In the current study the lesser prevalence of vitamin D among sick children than healthy children might be due to the fact that, most of the studies included for the sick children were conducted among malnourished children and tuberculosis children. Malnourished children were usually fed malnutrition treatment formula which have high vitamin $D[30]$ and this might mask the usual vitamin deficiency among children. Similarly, as tuberculosis is a granulomatous disease and in granulomatous diseases, activated mononuclear cells (particularly macrophages) in the lung and lymph nodes produce calcitriol from calcidiol independent of PTH[31-34]. This may increase the serum level of vitamin D among children with tuberculosis. Furthermore, some studies among HIV patients show that ART treatment drugs particularly, use of ritonavir or tenofovir, was associated with higher levels of 25OHD[35] and this might also be contributed for higher vitamin D among the sick groups.

From another systematic review and meta-analysis done to assess vitamin D deficiency in critically ill children in the other study, the pooled VDD event rate was 54.8 (95\% CI 45.463.9) and another study done in developing countries comparable to the current study also showed a higher pooled VDD rate of $64 \%$ with $95 \%$ CI 51-75\%)[36] which is much higher than the finding of the current study. This might be due to the nature of the participants of the studies, where the participants of the previous studies were critically ill children in ICU while those in the current studies were not ICU patients.

Finding of systematic review and meta-analysis conducted to assess vitamin D deficiency in Africa among the healthy children found that, the pooled prevalence was 49.07 with $95 \% \mathrm{CI}$ 
of (24.88-73.49) among newborns and 22.99 (12.03-36.14) among older children [37]. The pooled prevalence from this study is much lower than the finding from the current study. This might be due to the fact that, most of the studies included in the earlier meta-analysis were relatively from well-off countries and the studies also used random effect meta-analysis to report the pooled prevalence.

This study has attempted to put the burden of vitamin D deficiency among under-five children in sub-Saharan Africa using different segment of population based on health status of children. It has tried to delineate the difference in the burden of the problem among healthy and sick children. However, there are certain important limitation to be considered while using the findings of this study. One of the limitation of this study was that, all most all of the studies included in this review were considered different age groups of children and this resulted in difficulty of conducting subgroup analysis using the age group and other important factors.

\section{Conclusion}

In general, the pooled prevalence of vitamin D deficiency among under-five children in this study was found to be among the higher public health concern and there is significant variation in the prevalence from country to country. The pooled prevalence among the healthy children was found to be higher than the prevalence among the sick children. Correspondingly, the mean serum vitamin D level among the sick children was higher than among the healthy children.

\section{Declarations:}

Funding: This research received no external funding

Acknowledgements: We acknowledge the authors and participants of the included original studies in this systematic review and Meta analysis

Availability of data and materials: Data underlying the study is readily available can be obtained from the corresponding author on reasonable request.

Authors' contributions: "Conceptualization, M.F. and R.H.; methodology, M.F. and R.H.; software, M.F. and R.H.; validation, M.F. and R.H.; formal analysis, M.F., MD and R.H.; investigation, M.F., MD and R.H.; resources, M.F. and R.H.; data curation, M.F., MD and 
R.H.; writing — original draft preparation, M.F., MD and R.H.; writing—review and editing, M.F., MD and R.H.; visualization, M.F., MD and R.H.; supervision, M.F., MD and R.H.; project administration, M.F., and R.H.; funding acquisition, none. All authors have read and agreed to the published version of the manuscript

Conflict of interest: Authors declare no competing interests.

Consent for publication: Not applicable

Ethical approval: The procedures used in this study respects the scientific ethical rules.

\section{Reference}

[1] Viteri FE, Gonzalez H. Adverse outcomes of poor micronutrient in childhood and adolescence. Nutr Rev. 2002;60:S77-83.doi:10.1301/00296640260130795 n.d.

[2] Sight and life. Executive Report, The Global Hidden Hunger Indices and Maps: An Advocacy Tool for Action. Vitam Motion 2017:1-4.

[3] Bosomworth NJ. Mitigating epidemic vitamin D deficiency: the agony of evidence. Can Fam Physician 2011;57:16-20 n.d.

[4] Norman AW, Bouillon R. Vitamin D nutritional policy needs a vision for the future. Exp Biol Med (Maywood). 2010 Sep; 235(9):1034-45 n.d.

[5] Omar N, Mosaad Y. Vitamin D and immune system. Vitam Miner 2017; 6:151 n.d.

[6] Zisi D, Challa A, Makis A. The association between vitamin D status and infectious diseases of the respiratory system in infancy and childhood. Hormones 2019;18:35363. https://doi.org/10.1007/s42000-019-00155-z.

[7] Buonsenso D et al. Vitamin D levels in active TB, latent TB, non-TB pneumonia and healthy children: a prospective observational study. Fetal Pediatr Pathol 2018:1-11 n.d.

[8] Agarwal A et al. Vitamin D status in pediatric osteoarticular tuberculosis. J Clin Orthop Trauma 2015: 6(4):227-229 n.d.

[9] Dabla PK et al. Vitamin D deficiency among pediatric osteoarticular tuberculosis patients. J Clin Orthop Trauma 2016: 7(Suppl 2):147-149 n.d.

[10] Williams B, Williams AJ, Anderson ST. Vitamin D deficiency and insufficiency in 
children with tuberculosis. Pediatr Infect Dis J 2008: 27(10):941-942 n.d.

[11] Gray K et al. Vitamin d and tuberculosis status in refugee children. Pediatr Infect Dis J 2012: 31(5):521-523 n.d.

[12] Venturini E et al. Vitamin D and tuberculosis: a multicenter study in children. BMC Infect Dis 2014: 14:652 n.d.

[13] WHO. W.H.O., Global tuberculosis report 2013. 2013 https:// apps.who.int/iris/handle/10665/91355 n.d.

[14] Ludmir $\mathbf{J}$ et al. Vitamin D status in Botswana children under 2 years old with and without active tuberculosis. Am J Trop Med Hyg 2016: 94(5):971-974 n.d.

[15] Jubulis J et al. Modifiable risk factors associated with tuberculosis disease in children in Pune. India Int J Tuberc Lung Dis 2014: 18(2):198-204 n.d.

[16] Bachrach LK (2001) Acquisition of optimal bone mass inchildhood and adolescence.Trends Endocrinol Metab12,22-28. n.d.

[17] Rauch F (2007) Bone accrual in children: adding substance to surfaces. Pediatrics119, Suppl. 2, S137-S140. n.d.

[18] Mokhtar RR, Holick MF, Sempértegui F, Griffiths JK, Estrella B, Moore LL, et al. Vitamin D status is associated with underweight and stunting in children aged 6-36 months residing in the Ecuadorian Andes. Public Health Nutr 2018;21:1974-85. https://doi.org/10.1017/S1368980017002816.

[19] umar GT, Sachdev HS, Chellani Het al. Effect ofweekly vitamin D supplements on mortality, morbidity, andgrowth of low birthweight term infants in India up to age 6 months: randomised controlled trial. BMJ; 2011, 342, d2975 n.d.

[20] Gilbert-Diamond D, Baylin A, Mora-Plazas M, Marin C, Arsenault JE, Hughes MD, et al. Vitamin D deficiency and anthropometric indicators of adiposity in school-age children: A prospective study. Am J Clin Nutr 2010;92:1446-51. https://doi.org/10.3945/ajcn.2010.29746.

[21] Robinson SL, Ramirez-Zea M, Roman AVet al. Cor-relates and family aggregation of vitamin D concentrationsin school-aged children and their parents in nine Mesoamerican countries. Public Health Nutr 2017; 20, 2754-2765. n.d. 
[22] G. Wells, B. Shea, and D. OConnell, The Newcastle-Ottawa Scale (NOS) for assessing the quality of nonrandomised studies in meta-analyses, Ottawa Hospital Research Institute, Ottawa, Canada (2009) n.d.

[23] Ru“cker G, Schwarzer G, Carpenter JR, Schumacher M: Undue reliance on I2 in assessing heterogeneity may mislead. BMC Medical Research Methodology 2008, 8:79. https://doi.org/10.1186/1471-2288- 8-79 PMID: 19036172 n.d.

[24] Nabeta HW, Kasolo J, Kiggundu RK, Kiragga AN, Kiguli S. Serum vitamin D status in children with protein - energy malnutrition admitted to a national referral hospital in Uganda. BMC Res Notes 2015;8:1-8. https://doi.org/10.1186/s13104-015-1395-2.

[25] Hassam I, Kisenge R, Aboud S, Manji K. Association of vitamin D and diarrhoea in children aged less than five years at Muhimbili national hospital, Dar es Salaam : an unmatched case control study. BMC Pediatr 2019;19:1-9.

[26] Ludmir J, Mazhani L, Cary MS, Chakalisa UA, Pettifor JM, Molefi M, et al. Vitamin D Status in Botswana Children under 2 Years Old with and without Active Tuberculosis. Am J Trop Med Hyg 2016;94:971-4. https://doi.org/10.4269/ajtmh.150864.

[27] Sudfeld CR, Manji KP, Smith ER, Aboud S, Kisenge R, Fawzi WW, et al. Vitamin D Deficiency Is Not Associated With Growth or. JPGN 2017;65:467-74. https://doi.org/10.1097/MPG.0000000000001658.

[28] Naik AL, Rajan MG, Manjrekar PA, Shenoy MT, Shreelata S, Srikantiah RM, et al. Effect of DOTS Treatment on Vitamin D Levels in Pulmonary Tuberculosis. J Clin Diagnostic Res 2017;11.

[29] Prentice A, Schoenmakers I, Jones KS, Jarjou LMA, Goldberg GR. Vitamin D deficiency and its health consequences in Africa. Clin Rev Bone Miner Metab 2009;7:94-106. https://doi.org/10.1007/s12018-009-9038-6.

[30] WHO. Guidelines for an integrated approach to the nutritional care of HIV-infected children (6 months-14 years). 2009.

[31] Insogna KL, Dreyer BE, Mitnick M, et al. Enhanced production rate of 1,25dihydroxyvitamin D in sarcoidosis. J Clin Endocrinol Metab 1988; 66:72 n.d. 
[32] Mason RS, Frankel T, Chan YL, et al. Vitamin D conversion by sarcoid lymph node homogenate. Ann Intern Med 1984; 100:59 n.d.

[33] Adams JS, Singer FR, Gacad MA, et al. Isolation and structural identification of 1,25dihydroxyvitamin D3 produced by cultured alveolar macrophages in sarcoidosis. J Clin Endocrinol Metab 1985; 60:960 n.d.

[34] Adams JS, Sharma OP, Gacad MA, Singer FR. Metabolism of 25-hydroxyvitamin D3 by cultured pulmonary alveolar macrophages in sarcoidosis. J Clin Invest 1983; 72:1856 n.d.

[35] Yin M, Stein E. The effect of antiretrovirals on vitamin D. Clin Infect Dis 2011;52:406-8. https://doi.org/10.1093/cid/ciq169.

[36] McNally JD, Nama N, O’Hearn K, Sampson M, Amrein K, Iliriani K, et al. Vitamin D deficiency in critically ill children: A systematic review and meta-analysis. Crit Care 2017;21:1-13. https://doi.org/10.1186/s13054-017-1875-y.

[37] Mogire RM, Mutua A, Kimita W, Kamau A, Bejon P, Pettifor JM, et al. Prevalence of vitamin D deficiency in Africa: a systematic review and meta-analysis. Lancet Glob Heal 2020;8:e134-42. https://doi.org/10.1016/S2214-109X(19)30457-7. 